\title{
Henri Nonn, L'Alsace actuelle
}

Presses Universitaires de Strasbourg, 204 p.

\section{Raymond Woessner}

\section{(2) OpenEdition}

\section{Journals}

Electronic version

URL: http://journals.openedition.org/rge/5612

DOI: $10.4000 /$ rge.5612

ISSN: 2108-6478

\section{Publisher}

Association des géographes de l'Est

\section{Printed version}

Date of publication: 1 October 2015

ISSN: 0035-3213

Electronic reference

Raymond Woessner, « Henri Nonn, L'Alsace actuelle », Revue Géographique de l'Est [Online], vol.55 / n०3-4 | 2015, Online since 14 October 2015, connection on 23 September 2020. URL : http:// journals.openedition.org/rge/5612 ; DOI : https://doi.org/10.4000/rge.5612

This text was automatically generated on 23 September 2020.

Tous droits réservés 


\section{Henri Nonn, L'Alsace actuelle}

Presses Universitaires de Strasbourg, 204 p.

Raymond Woessner

\section{REFERENCES}

Henri Nonn, L'Alsace actuelle, Presses Universitaires de Strasbourg, $204 \mathrm{p}$. 
1 A l'heure où la Région Alsace (1955-2015) commence à se fondre dans la grande région Alsace-Champagne-ArdennesLorraine (ACAL), l'ouvrage de Henri Nonn arrive à point nommé. Comment fonctionne le territoire alsacien, quelle est sa personnalité, et cela après plusieurs décennies de mondialisation? L'ouvrage appartient à la lignée de l'université de Strasbourg qui, dès les années 1960, avait contribué à réinventer la géographie régionale avec Étienne Juillard (1914-2006), Jean Tricart (1920-2003), Michel Rochefort (1927-2015)... Henri Nonn invite d'abord à une lecture géohistorique depuis l'après-guerre, puis explique les logiques spatiales à partir de la métropolisation, et s'interroge enfin sur les modes de gouvernance. Il donne un

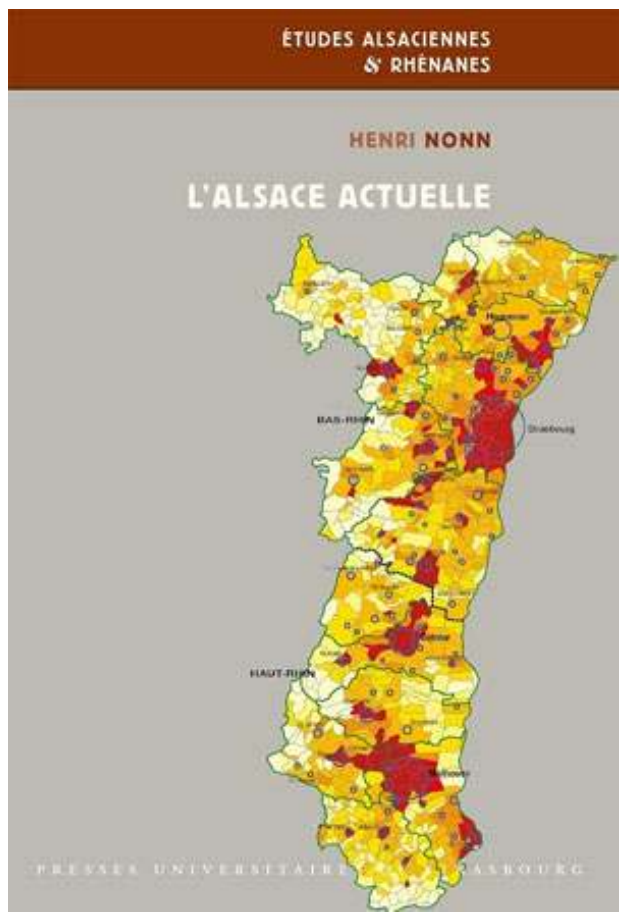
sens à l'histoire sous la forme d'une « dynamique de progrès collectif » désormais inscrit dans la durabilité.

2 La partie I montre comment le développement de l'Alsace s'est fait de manière exogène, "sous dépendance » des capitaux français et internationaux, ce qui avait été largement souhaité par des responsables alors soucieux de mettre en valeur une position rhénane. En effet, au début des Trente Glorieuses, les " paysages soignés » et « coquets» cachaient une structure agricole fragile avec de trop petites exploitations, une «mosaïque » d'industries anciennes et des banques absorbées par des réseaux nationaux avec à la clé un «déclin » du «capitalisme régional». Mais l'intégration européenne et la modernisation des infrastructures, soutenue par la planification française, impulsent une vigoureuse croissance fondée sur l'internationalisation. L'agriculture devient maïsiculture et viticulture (avec aujourd'hui $30 \%$ des crémants élaborés en France). Le phénomène des travailleurs frontaliers vers Karlsruhe et Bâle prend corps dans les années 1960. Au total, la prospérité masque une faiblesse du fait de l'importance considérable des emplois de production, alors que le tertiaire supérieur reste en retrait. Alertée par un rapport du CESA en 2003, la Région promeut l'innovation (avec le réseau BioValley, par exemple). Elle est par ailleurs soucieuse de durabilité (TER, plans paysagers, gestion de l'eau...). Après un lent effritement, la crise de 2008 marque la fin d'une époque avec la destruction de nombreux emplois, en particulier dans l'industrie.

3 La partie II évoque "le nouveau paradigme » articulé entre les échelles globale et locale, où la métropolisation est la clé de voûte du développement, à condition de pouvoir répandre ses effets dans toute la région. Dans ce jeu, les atouts de Strasbourg sont les suivants : une ville-porte avec de bonnes infrastructures, une université de renom et la présence des institutions européennes. Tramway et tourisme sont des marqueurs positifs. Mais la recherche manque de lisibilité autour d'un pôle qui serait emblématique. À Mulhouse, l'aéroport constitue un atout essentiel; ses musées techniques sont réputés; mais la ville est toujours un « réceptacle » pour l'industrie et 
non une "inductrice d'activités ». La ville a renoncé à la Métropole Rhin-Rhône et cherche depuis 2011 des synergies avec Strasbourg. Quant aux villes moyennes, elles apparaissent dans leur rhénanité avec la présence de fonctions supérieures et de services diversifiés. Les effets d'entraînement de Bâle et Strasbourg sont évidents, encore que leurs fonctions supérieures ne traversent guère la frontière nationale et que l'Alsace fonctionne d'abord pour elles comme une sorte de périphérie heureuse.

La partie III interroge le futur à l'aune des acquis. L'auteur est un peu dubitatif quant au classement national de Strasbourg en termes d'emplois métropolitains car, bien plus qu'ailleurs en France, une partie de cette ressource est établie à proximité mais non dans son aire urbaine. Par ailleurs, la dynamique socio-démographique alsacienne s'essouffle d'un point de vue quantitatif, avec une croissance de la population fondée sur le seul solde naturel et qui va en se ralentissant. De fait, l'intégration des « cadres allogènes » - discrets par leur nombre dans les bilans statistiques - devient un élément déterminant pour l'emploi et la création de richesses, la demande de mobilités et de logements verts comme celle d'événements dans le but de "faire métropole ». Dans les banlieues et dans le périurbain, la promotion d'un urbanisme qui ne serait pas seulement de l'urbanisation est complexe à mettre en œuvre, notamment du fait du mille-feuille administratif et politique à la complexité croissante. C'est pourquoi est apparue légitimement la question de la fusion des deux départements et de la création d'une collectivité unique, un projet qui avait échoué faute de "clarté ", de "préjugés ou inerties». À présent, la création d'une grande région vient «compliquer les perspectives » en ajoutant une échelle supplémentaire.

5 Pour l'auteur, les politiques de soutien à l'innovation sont une priorité car «le développement régional [est] à revigorer ». Il invite à un rapprochement entre les institutions et les entreprises pour une "endogénéisation » dans une région où les forces sont "assez bien réparties" avec de fortes "aménités régionales/locales ». L'auteur dénonce le «saupoudrage » et propose d'accentuer l'effort sur deux pôles, "santé-médicaments-biotechnologies» et "culture et créativité ». Le carrefour logistique apparait lui aussi comme une opportunité. Finalement, la frontière nationale reste un facteur perturbant car elle freine les jeux d'acteurs et les intégrations au sein du Rhin supérieur. "Sont en tout cas indispensables des ajustements de gouvernance ». Et, préoccupation récurrente de l'auteur, une clé importante du développement se situe dans l'organisation et la promotion des mailles fines du territoire alsacien.

6 L'ouvrage est complété par une belle annexe cartographique en couleur, avec un croquis de 2005 très instructif sur la convergence des trajectoires des métropoles régionales en France, que l'on se désespérait de voir publier un jour. Le glossaire est instructif et la bibliographie, séduisante. Malgré la densité de l'information et la consistance des idées, il y a en fin de compte un peu de frustration pour le lecteur qui voudrait imaginer les contours de l'Alsace du futur, ce qui, il est vrai, n'était pas le titre de l'ouvrage. 